\title{
Pathophysiologische Grundlagen der mechanischen Beatmung
}

\section{Köhler ${ }^{1}$ \\ M. Pfeifer ${ }^{2}$ \\ C. Criée ${ }^{3}$}

\section{Pathophysiological Basis of Mechanical Ventilation}

\section{Zusammenfassung}

Eine Beatmung ist immer dann erforderlich, wenn die eigene Ventilation nicht mehr ausreicht. Dieses zeigt sich typischerweise in einer Hyperkapnie. Die Hypoxämie folgt hier meist nur sekundär aufgrund der Hypoventilation. Ursache ist in der Regel eine überlastete Atemmuskulatur (Atempumpe), meist durch eine akute oder chronische Erkrankung. Bei isolierter Hypoxämie ist eine Beatmung nur dann indiziert, wenn der Sauerstoffgehalt ( = Sauerstoffsättigung $\times \mathrm{Hb} . \times 1,39$ ) unter eine kritische Schwelle sinkt oder infolge der fortschreitenden Grunderkrankung (z.B. Pneumonie) und zusätzlicher Überlastung die Erschöpfung der Atemmuskulatur droht. Neben dem positiven Effekt auf den Gasaustausch kann Beatmung an sich insbesondere durch zu hohe Drucke, Volumina oder Sauerstoffkonzentration zur Schädigung des Lungenparenchyms führen. Durch lungenprotektive Beatmungsstrategien lassen sich diese Schäden weitgehend vermeiden. Hierbei muss im Einzelfall geprüft werden, nach welchen Parametern beatmet werden soll. Ist der Leitparameter nicht wie üblich der Sauerstoffpartialdruck, sondern der Sauerstoffgehalt, kommt es in der Regel zu einer deutlich schonenderen Beatmung. Eine Ausnahme hiervon ist das kardial bedingte Lungenödem, da die Beatmung hier durch den Beatmungsdruck und den PEEP zur deutlichen Verbesserung der Diffusion, Senkung der Preload und eine Abnahme der Atemarbeit führt. Ein entscheidender Fortschritt in der Beatmung insbesondere von schweren Akutfällen wurde in den letzten Jahren durch Erweiterung der Beatmungszugänge, d.h. nicht-invasive Alternativen zum Beatmungstubus erreicht. Die Maskenbeatmung ist insbe-

\section{Abstract}

Mechanical ventilation is required if ventilatory insufficiency is present. This is typically indicated by hypercapnea. Hypoxemia occurs secondary to hypoventilation. Usually overload of the respiratory muscles (ventilatory pump) will be the underlying mechanism, for the most part caused by acute or chronic disease. In case of sole hypoxemia mechanical ventilation will only be indicated if the oxygen-content (equals oxygensaturation $\times$ haemoglobin $\times 1.39$ ) drops below a critical threshold or if ventilatory pump failure is immanent on account of the underlying disease (eg. pneumonia). The background of our recommendations is to avoid potential damage caused by mechanical ventilation. Especially high inspiratory pressures and oxygen concentrations can be harmful to the lung. Therefore every case has to evaluated for individual target parameters of ventilation. The use of the oxygen-content instead of the arterial oxygen pressure as the target parameter will usually lead to a more careful ventilation. Cardiogenic pulmonary oedema is an exception to this rule since inspiratory positive pressure and PEEP will result in improved diffusion as well as reduction of preload and work of breathing. In recent years progress has been made on the field of ventilation access especially in severe and acute cases. Non-invasive ventilation is superior to invasive ventilation in patients with exacerbated COPD since it improves outcome effectively. This is being caused by a decline in ventilator associated pneumonias, most likely because non-invasive ventilation allows patients to clear their secretions by coughing, resulting in improved lung clearance. Controlled ventilation allows optimal unloading of the respi-

${ }^{1}$ Krankenhaus Kloster Grafschaft, Schmallenberg

${ }^{2}$ Klinik Donaustauf, Universität Regensburg, Donaustauf

${ }^{3}$ Evangelisches Krankenhaus Göttingen-Weende, Bovenden

Korrespondenzadresse

Prof. Dr. med. Dieter Köhler · Ärztlicher Direktor · Krankenhaus Kloster Grafschaft ·

Annostr. 1·57292 Schmallenberg ·E-mail: d.koehler@fkkg.de

Bibliografie

Pneumologie 2006; 60: 100-110 @ Georg Thieme Verlag KG Stuttgart · New York

DOI 10.1055/s-2005-919155

ISSN 0934-8387 
sondere bei exazerbierter COPD der invasiven Beatmung bezüglich des Outcome drastisch überlegen. Dies wird insbesondere durch einen Rückgang der ventilator-assoziierten Pneumonie verursacht, vermutlich weil die Patienten unter Maske noch abhusten können, was die Lungenreinigung deutlich verbessert. Eine optimale Entlastung der durch die Erkrankung überlasteten Atemmuskulatur wird durch eine kontrollierte Beatmung ermöglicht. Der kontrollierte Beatmungsmodus lässt sich aber im Akutfall in der Regel nur mit Sedation erreichen. Eine assistierte Beatmung bessert zwar den Gasaustausch, führt jedoch nur zu einer mäßigen Entlastung der Atemmuskulatur, was ihre Rekompensation verzögert. Eine dauerhafte kontrollierte Beatmung unter Sedation über einen längeren Zeitraum (Tage) benötigt zwischengeschaltete kurze Phasen mit assistierter- oder Spontanatmung, um eine Atrophie der Atemmuskulatur zu verhindern. In der Übersicht werden die Hintergründe zwischen Sauerstoffangebot und -verbrauch insbesondere unter Berücksichtigung der Atempumpeninsuffizienz und ihrer Beeinflussbarkeit durch verschiedene Beatmungsverfahren dargestellt. ratory muscles which have been overloaded by the underlying disease. Application of a controlled ventilation mode in acute disease will usually require some kind of sedation. Assisted ventilation will result in improved gas exchange but only incomplete unloading of respiratory muscles and therefore delayed restitution. Permanent controlled ventilation under sedation for a prolonged period (days) requires intermittent periods of assisted- or spontaneous breathing in order to avoid atrophy of the respiratory muscles. This review summarizes background information on the nature of the derangement, the relation between oxygen supply and consumption under special consideration of respiratory muscle insufficiency and impact of different ventilation modes.

\section{Einleitung}

Ohne die Möglichkeiten der künstlichen Beatmung wäre sowohl die Behandlung vieler akuter Erkrankungen nicht mehr denkbar, als auch komplexe operative Eingriffe nicht durchführbar. Etwa ein Drittel aller Patienten auf Intensivstationen ist beatmet [1]. Ursache ist eine primäre oder sekundäre Störung des Gastransportes (Gasaustausch und Ventilation) aufgrund unterschiedlicher Erkrankungen. Die mechanische Beatmung sichert im Wesentlichen die alveoläre Ventilation und ermöglicht durch Applikation eines erhöhten intraalveolären Dauerdrucks (PEEP) die Prävention und/oder die Besserung von Atelektasen und damit die Reduktion eines Shuntvolumens [2,3].

Auch wenn eine moderne Medizin ohne maschinelle Beatmung nicht mehr denkbar ist, sind die potenziellen Gefahren einer artifiziellen Ventilation der Lunge nicht gering und müssen von jedem, der eine Beatmung durchführt, gekannt und berücksichtigt werden.

Allein die Umkehrung der Druckverhältnisse vom negativen inspiratorischen Druck während der Spontanatmung zum positiven Druck unter maschineller Beatmung führt zu einer unphysiologischen Belastung der Atemwege, des Lungenparenchyms und des Herzkreislaufsystems. Hohe Beatmungsdrucke verletzen mechanisch durch Überdehnung (shear stress, Volutrauma, Barotrauma) das Alveolarepithel und induzieren somit eine von der Grunderkrankung unabhängige Schädigung der Lunge mit einer schweren Inflammationsreaktion [4]. Die Gefahr von hohen Beatmungsdrücken ist immer dann gegeben, wenn die Beatmungstherapie allein nach Blutgaswerten gesteuert wird. Nicht selten werden die Patienten unnötigerweise „überbeatmet“, um „normale“ Blutgaswerte für $\mathrm{PaO}_{2}$ und $\mathrm{PaCO}_{2}$ zu erreichen. Wenn dazu noch inadäquat hohe inspiratorische Sauerstoffkonzentrationen $(>50 \%$ ) über längere Zeit gewählt werden, besteht die Gefahr einer weiteren toxischen Schädigung der Lunge [5 - 7].
Darüber werden immer noch die Langzeitschäden der Intubationsbeatmung wie Kehlkopfveränderung, Trachealstenosen, frakturierte Knorpelspangen mit Instabilität usw. zu wenig beachtet - sicherlich auch deshalb, weil der Intensivmediziner diese erst nach Wochen bis Monaten auftretenden Spätkomplikationen sehr selten zu Gesicht bekommt [8,9]. Die Intubation begünstigt in Verbindung mit der Sedation durch die Unterbrechung der Lungenreinigung (aufgehobene mukoziliäre Clearance und fehlende Hustenmöglichkeit) die ventilatorassoziierte Pneumonie $[10,11,13]$. Hohe Beatmungsdrucke bzw. hoher PEEP können zudem das Herzminutenvolumen kritisch reduzieren [13 - 15], was zu einer hämodynamischen Instabilität und zu weiteren Komplikationen führen kann.

Die Kunst des Intensivmediziners besteht darin, die Indikation zur Beatmung richtig zu stellen und diese angepasst an das Krankheitsbild adäquat einzusetzen. Das ist ohne Kenntnis der Grundlagen des Gastransportes, der Atempumpe, der Lungenperfusion sowie deren Interaktion mit der maschinellen Beatmung nicht möglich.

Das wichtigste Ziel in der Intensivmedizin ist es, die Grundkrankheit zu behandeln und die nicht betroffenen Organe in der Funktion zu erhalten bzw. zu stabilisieren. Häufig werden aber Adaptionsvorgänge des Organismus falsch interpretiert und führen nicht selten zu Überreaktionen von Seiten der behandelnden Ärzte mit nicht seltenen negativen Auswirkungen auf den Verlauf. Ein typisches Beispiel ist die unkritische Anwendung von Katecholaminen, die meist zu hoch dosiert oder kombiniert mit anderen kreislaufwirksamen Medikamenten verabreicht werden. Die durchaus oft sinnvolle Gegenregulation des Organismus von den Folgen der Erkrankung zu trennen, ist mitunter nicht einfach und erfordert profunde Kenntnisse der pathophysiologischen Zusammenhänge. Die Antwort des Organismus auf die akute Erkrankung führt nicht selten dazu, dass die messbaren physiologischen Variablen wie Blutgase, Blutdruck, Herzminutenvolumen, Atemzugvolumen, Atemfrequenz scheinbar in den 
pathologischen Bereich abrutschen und deshalb zu entsprechenden Interventionen führen. Diese Interventionen können wiederum zu einer zusätzlichen Belastung des Organismus führen und sind nicht selten für eine erhöhte Mortalität verantwortlich. Wichtige Anpassungen an die hypoxische Erkrankungssituation entziehen sich den einfachen Messmethoden, wie Blutumverteilung zwischen den Organen [16,17], Änderung der Sauerstoffbindungskurve durch Änderung der 2,3 DPG-Konzentration [18,19], Expression von sauerstoffresistenten Isoenzymen der Atmungskette [20-22] oder Transportänderungen der Energieträger in die Zelle [23-25], obwohl sie für die Behandlung des kritisch Kranken wesentliche Informationen wären.

Die meisten Fehler werden - wie erwähnt - durch eine Überbewertung der Blutgase gemacht. Häufig wird versucht, ein supranormales Sauerstoffangebot einzustellen. Hierzu müssen meist hohe inspiratorische Sauerstoffkonzentrationen eingesetzt werden. Ein Beispiel für diese Praxis ist die bekannte Arbeit von Gattinoni u. Mitarb. [26] zur Frage des Einflusses der Bauchlagerung auf die Mortalität bei ARDS. Beide Patientengruppen hatten eine 30-Tage-Mortalität von etwa $50 \%$. Hierbei wurden $\mathrm{FiO}_{2}$-Werte um $70 \%$ eingestellt, um einen Sauerstoffpartialdruck von über $85 \mathrm{~mm} \mathrm{Hg}$ zu erreichen. Dieser stieg in Bauchlage auf etwa $100 \mathrm{~mm} \mathrm{Hg}$ an. Diese hohen Sauerstoffpartialdruckwerte haben keinen relevanten Einfluss mehr auf die tatsächliche Sauerstoffversorgung des Organismus, die sich im Wesentlichen im Sauerstoffgehalt widerspiegelt. Deswegen überrascht es nicht, dass beide Behandlungsgruppen keine Unterschiede zeigten.

\section{Respiratorische Insuffizienz}

Bei der Klassifikation der respiratorischen Insuffizienz wird üblicherweise von einem hyperkapnischen bzw. einem hypoxischen Versagen gesprochen [27]. Beides stellt in seiner schweren Form eine Indikation zur Beatmung dar. Die Klassifikation nach den Blutgasen hat jedoch den Nachteil, dass bereits eine Folge der Organstörung als Einteilungskriterium genommen wird. Dies kann jedoch insbesondere bei der Hypoxämie in die Irre bzw. zu falschen therapeutischen Maßnahmen führen. Es ist besser, das jeweils erkrankte Organsystem in den Vordergrund der Einteilung zu stellen, dargestellt in Abb.1.

Liegt eine Erkrankung des Lungenparenchyms vor, sei es durch Teilausfall wie bei einer Pneumonie oder durch Diffusionsstörung infolge akuter Linksherzinsuffizienz, dann ist die Sauerstoffaufnahme die gestörte Leitgröße. Diese Patienten sind durch kompensatorische Steigerung der Ventilation typischerweise hypokapnisch. Die Behandlung besteht hier - neben der Therapie der Grunderkrankung - in einer Verhinderung einer lebensbedrohlichen Hypoxämie. Eine Korrektur der Hypoxämie bis zum Normalwert ist dabei meist nicht erforderlich, da sonst das Lungenparenchym durch die dazu oft erforderlichen hohen Beatmungsdrucke und Sauerstoffkonzentration sekundär geschädigt wird.

Ist hingegen die Ventilation gestört, so steht eine Überlastung der inspiratorischen Atemmuskulatur im Vordergrund. Die Atemmuskulatur wird mit dem Thorax funktionell sinnvollerweise auch als Atempumpe zusammengefasst. Der Muskelanteil
Hypoxämisches Versagen=Lungenparenchymversagen

\section{$\mathrm{PaO}_{2} \downarrow$ \\ $\left(\mathrm{PaCO}_{2} \downarrow-\mathrm{n}\right)$}

Beispiele:

reduzierte Gasaustauschfläche: Emphysem, Pneumonie

erhöhte Diffusionsstörung: Fibrose, Lungenstauung

Respiratorische-Global-Insuffizienz

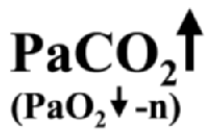

\section{Hyperkapnisches Versagen=Atempumpversagen}

Beispiele:

erhöhte Last: COPD, schwerer Asthmaanfall, Skoliose, Post TB

reduzierte Kapazität: neuromuskuläre Erkrankungen, Postpolio, Muskelatrophie

Abb. 1 Einteilung der respiratorischen Insuffizienz in hypoxämisches und hyperkapnisches Versagen. Bei beiden Gruppen kommt es zur Hypoxämie, die nur beim hypoxämischen Versagen primär, beim hyperkapnischen Versagen infolge der Hypoventilation jedoch sekundär ist. Näheres siehe Text.

besteht beim Gesunden etwa zu $90 \%$ aus dem Zwerchfell. Akute Überlastungen durch inadäquate Atemarbeit zur Überwindung der viskösen Widerstände (Druckarbeit) kommen z.B. durch einen Asthmaanfall oder eine exazerbierte COPD vor. Eine Volumenbelastung der Atempumpe entsteht durch stark gesteigerte Ventilationen wie z. B. bei fortschreitender Pneumonie. Daneben sind direkte Erkrankungen der Muskulatur oder der versorgenden Nerven möglich, wie bei erworbener Myopathie oder progredienten angeborenen neuromuskulären Erkrankungen. Bei zunehmender Überlastung kommt es zu einer verstärkten Hypoventilation, die auch als Protektion vor einer lebensbedrohlichen Erschöpfung gesehen werden kann, mit der Folge einer Hyperkapnie - aber auch einer sekundären Hypoxämie [28-31]. Bei diesen Patienten ist es üblicherweise nicht erforderlich, die Hypoxämie zu korrigieren. Mit der Behandlung der Hyperkapnie (in der Regel durch Beatmung) bessert sich die Hypoxämie automatisch.

\section{Sauerstoffangebot}

Eine wichtige Größe, die der Organismus konstant zu halten versucht, ist die Sauerstofftransportkapazität bzw. das Sauerstoffangebot $\left(\mathrm{DO}_{2}\right)$, das in Ruhe beim Gesunden etwa $250 \mathrm{ml} / \mathrm{min}$ beträgt. Unter körperlicher Belastung kann die $\mathrm{DO}_{2}$ bis auf das 20fache gesteigert werden. Die Sauerstofftransportkapazität stellt das Produkt aus Herzzeitvolumen und Sauerstoffgehalt $\left(\mathrm{CaO}_{2}\right)$ dar - eine Größe, die im klinischen Alltag zu wenig Beachtung findet. Der $\mathrm{CaO}_{2}$ selbst kann aus der Sauerstoffsättigung $\left(\mathrm{SaO}_{2}\right) \times$ Hämoglobin $\times$ der Hüfnerschen Zahl 1,39 errechnet werden (Abb. 2). Der Sauerstoffgehalt ist somit das Sauerstoffvolumen, das in $100 \mathrm{ml}$ Blut enthalten ist $\left(\mathrm{mlO}_{2} / 100 \mathrm{ml} \mathrm{Blut}=\right.$ Volumenprozent). Der Gehalt an Sauerstoff im Blut bestimmt gemeinsam mit dem Sauerstoffbindungsverhalten des Hämoglobins (Sauerstoffbindungskurve) den Sauerstoffpartialdruck $\left(\mathrm{PaO}_{2}\right)$. Der Sauerstoffpartialdruck ist für die Sauerstoffversorgung des Organismus nur eine tertiäre Größe, besitzt aber als klinische Kenngröße eine wichtige Bedeutung, da er sich rasch bei Funktionsstörungen ändert. 
Sauerstoffangebot: $\mathrm{DO}_{2}$ Sauerstoffaufnahme: $\mathrm{VO}_{2}$

\section{$\mathrm{DO}_{2}=$ Cardiac output $\mathrm{x}$ art. Sauerstoffgehalt}

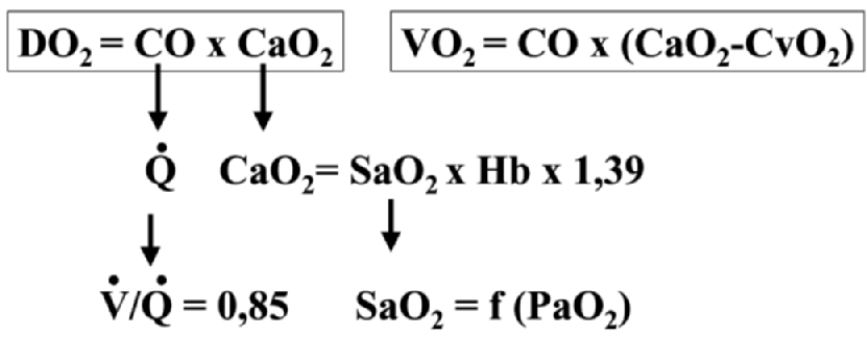

Abb. 2 Entscheidende Größen des Sauerstofftransportes. Das Sauerstoffangebot $\left(\mathrm{DO}_{2}\right)$ wird vom Organismus entsprechend des Bedarfs angepasst und geregelt. Es besteht aus der Zahl der Sauerstoffmoleküle (Sauerstoffgehalt) und der Perfusion (cardiac output). Da die Ventilation der Perfusion angepasst und ebenfalls geregelt wird (Ventilations-/Perfusionsquotient V/Q), muss die Ventilation ebenfalls dem Sauerstoffangebot angeglichen werden. Der Sauerstoffgehalt ist damit die primäre Größe für die Sauerstoffversorgung. Sättigung und vor allen Dingen Sauerstoffpartialdruck sind abgeleitete Parameter. Die Sauerstoffaufnahme $\mathrm{VO}_{2}$ (oder Extraktionsrate) wird durch die Differenz des arteriell-venösen Sauerstoffgehaltes bestimmt.

Der Kranke steigert seine Sauerstofftransportkapazität entsprechend dem gesteigerten Sauerstoffverbrauch, der durch die akute Erkrankung bedingt wird. Vergleichbar mit der körperlichen Belastung beim Gesunden reagiert der kranke Organismus in erster Linie mit einer Erhöhung des Herzzeitvolumens, um dem gesteigerten Sauerstoffbedarf gerecht zu werden. Dazu muss die Ventilation entsprechend gesteigert werden, um das Ventilations-/Perfusionsverhältnis konstant zu halten. Ansonsten käme es zur Hypoxämie. Eine weitere Steigerung der Ventilation bringt hingegen nichts, da der Sauerstoffgehalt dadurch nicht mehr zunehmen kann. Normalerweise ist das $\mathrm{DO}_{2}$ ca. 1,5 $\times$ höher als der Sauerstoffverbrauch $\left(\mathrm{VO}_{2}\right)$ [32]. Erhöht man beim Gesunden z. B. durch Sauerstoffgabe und Steigerung des Herzminutenvolumens mit Katecholaminen das $\mathrm{DO}_{2}$, so hat das in weiten Grenzen keinen Einfluss auf den $\mathrm{VO}_{2}[33-35]$.

Der Organismus hat in kritischen Situationen die Möglichkeit, die Sauerstoffextraktionsrate $\left(\mathrm{VO}_{2}\right)$ der Organe zu beeinflussen. Auf diese Möglichkeit greift er jedoch immer erst sehr spät zurück, weswegen eine Abnahme der $\mathrm{VO}_{2}$ prognostisch immer ein ungünstiges Zeichen ist [35].

Die Kenntnis der Formel zur Berechnung der Sauerstofftransportkapazität und das Wissen, dass die Sauerstofftransportkapazität möglichst lange konstant gehalten wird, erlauben die Erklärung zahlreicher Phänomene auf der Intensivstation. Liegt z. B. eine Anämie vor, so kommt es zur kompensatorischen Erhöhung des Herzminutenvolumens, um das $\mathrm{DO}_{2}$ konstant zu halten. Die Ventilation in dieser Formel (s. Abb. 2) ist indirekt über das Ventilations-/Perfusionsverhältnis an die Perfusion gekoppelt. Der Organismus versucht daher ebenfalls, die Ventilation der Perfusion anzupassen, so dass das Verhältnis von 0,85-1 etwa gleich bleibt. So wird bei einer Anämie nicht nur das Herzzeitvolumen zunehmen [36], sondern auch die Ventilation, was eine zusätzliche Last für die Atempumpe darstellt. Werden bei Patienten mit

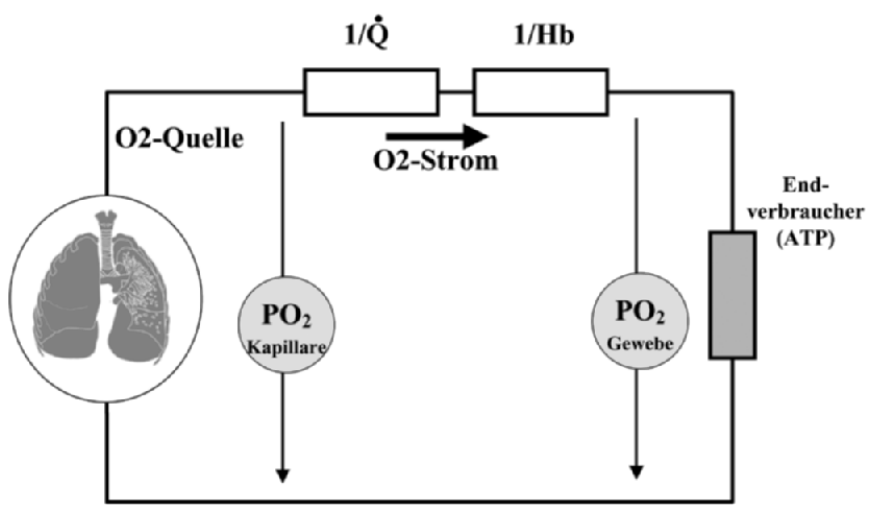

Abb. 3 Darstellung des Sauerstofftransports vor der Aufnahme über die Lunge $\left(\mathrm{O}_{2}\right.$-Quelle) bis zur Utilisation im Gewebe. Die Abb. zeigt die hintereinandergeschalteten Transportwege in Form eines Ersatzschaltbildes. Der Sauerstoffdruck wird bestimmt über die Perfusion (1/Q) und den Hämoglobingehalt (1/Hb). Daraus ergibt sich der Sauerstoffpartialdruck in der Peripherie. Eine niedrige Perfusion (indirekt dargestellt als hoher Widerstand) führt zu einem Sauerstoffpartialdruckabfall im Gewebe. Das gleiche gilt für ein niedriges Hämoglobin.

Anämie und respiratorischer Insuffizienz Erythrozytenkonzentrate substituiert, kommt es daher nicht nur zu einer Normalisierung des Herzzeitvolumens sondern auch zu einer Reduktion der Ventilation, was eine Entlastung der Atempumpe bedingt [37]. Die Reduktion der Ventilation führt zu einer Entlastung der Atemmuskulatur, so dass mitunter beim kritisch kranken Patienten auch schon bei leichter Anämie durch eine Bluttransfusion eine Beatmung vermieden oder das Weaning erleichtert werden kann [38]. Als Folge der reduzierten Ventilation können sich die Blutgase formal leicht verschlechtern d.h. $\mathrm{pCO}_{2}$ steigt und $\mathrm{pO}_{2}$ fällt etwas, dem Patienten aber geht es insgesamt klinisch besser. Dieses Beispiel zeigt sehr anschaulich, wie wichtig es ist, die pathophysiologischen Zusammenhänge zu kennen.

In Abb. 3 sind zur Veranschaulichung die einzelnen Kompartimente des Sauerstofftransportes in einem Ersatzschaltbild dargestellt. Sauerstoffquelle ist hier die Lunge mit der Ventilation, die den $\mathrm{PaO}_{2}$ in den Lungenkapillaren bestimmt. Zwei Leitwerte bestimmen dann den Transport in das Kapillarbett: die Perfusion und das Hämoglobin mit seiner Sauerstoffbindung. Der letzte Weg des Sauerstoffs vom Hämoglobinmolekül zum Mitochondrium ist ein Diffusionsvorgang, der bestimmt wird durch die Sauerstoffpartialdruckdifferenz zwischen dem hämoglobingebundenen Sauerstoff und dem intrazellulären Sauerstoff [32]. Dieser periphere $\mathrm{PaO}_{2}$ wird aber im Wesentlichen durch den $\mathrm{CaO}_{2}$ bestimmt. Die physikalische Löslichkeit des Sauerstoffs im Blut spielt quantitativ kaum eine Rolle. Eine Ausnahme von dieser Regel ist eine gestörte Perfusion in der Peripherie, wie sie typischerweise postoperativ im Wundbereich nach Durchtrennung der Kapillaren vorkommt. Hier reduziert ein erhöhter Anteil an physikalisch gelöstem Sauerstoff die Wundinfektion, vermutlich durch Abnahme des anaeroben Milieus im schlecht durchbluteten Bereich [39]. Ein erhöhter Anteil des gelösten Sauerstoffs wird durch eine Anhebung des $\mathrm{FiO}_{2}$ erreicht. 
Die für den Sauerstofftransport zentrale $\mathrm{Größe} \mathrm{CaO}_{2}$ wird in der Praxis auf den Intensivstationen noch zu wenig beachtet. Die Beziehungen zwischen den Größen $\mathrm{CaO}_{2}, \mathrm{SaO}_{2}$ und $\mathrm{PaO}_{2}$ sind in Abb. 4 noch einmal anschaulich dargestellt. Eine gesunde Frau mit einem Hämoglobin im unteren Grenzbereich hat bei einer $\mathrm{SaO}_{2}$ von $95 \%$ einen Sauerstoffgehalt von $14,8 \mathrm{ml} \mathrm{O}_{2} / \mathrm{ml}$ Blut. Legt man den gleichen Sauerstoffgehalt für einen Patienten mit schwerer COPD und chron. Hyperkapnie zugrunde, so hat er infolge seiner reaktiven Polyglobulie ( $\mathrm{Hb} 18 \mathrm{~g} \%$ ) eine $\mathrm{SaO}_{2}$ von $64 \%$ entsprechend einem $\mathrm{PaO}_{2}$ von $34 \mathrm{~mm} \mathrm{Hg}$. Dieser Patient ist hypoxämisch, jedoch nicht hypoxisch in der Peripherie.

Ein Nomogramm in Abb. 5 stellt die Beziehung zwischen $\mathrm{SaO}_{2}$ und $\mathrm{CaO}_{2}$ in Abhängigkeit von verschiedenen Hämoglobinkonzentrationen nochmals dar. Rautenförmig ist etwa der Normalbereich angegeben. Ein kritischer unterer Grenzwert für $\mathrm{CaO}_{2}$ ist nicht gesichert und lässt sich nur aufgrund indirekter Daten aus der Literatur ermitteln, z. B. aus Tierversuchen [40-44], aus Untersuchungen an freiwilligen Probanden $[45,46]$ oder an Patienten mit schwerer Anämie [47], sowie aus Ergebnissen der Höhenmedizinforschung [48-50]. Entscheidend ist, ob der Patient aus voller Gesundheit heraus erkrankt oder eine chronische respiratorische Insuffizienz vorliegt, die schon zu Kompensationsmechanismen der chron. Hypoxämie geführt hat. Für einen bisher gesunden Patienten ohne kardiale Einschränkung kann ein Wert unter 7-8 $\mathrm{mlO}_{2} / 100 \mathrm{ml}$ Blut als kritisch angesehen werden, während bei Patienten, die chronisch an eine Hypoxämie adaptiert sind (z.B. schwere COPD, Lungenfibrose), Werte ab kleiner 5-6 $\mathrm{mlO}_{2} / 100 \mathrm{ml}$ Blut gefährlich werden. Rechnet man einen $\mathrm{CaO}_{2}$ von 5-6 $\mathrm{mlO}_{2} / 100 \mathrm{ml}$ Blut auf den resultierenden $\mathrm{PaO}_{2}$ im Nomogramm um, so ist man erstaunt, wie hypoxieresistent der Organismus ist. Die Berücksichtigung des $\mathrm{CaO}_{2}$ führt bei der Indikation einer Beatmung bzw. für die Einstellung der Parameter zu einem differenzierteren Vorgehen als die alleinige Betrachtung des $\mathrm{PaO}_{2}$. Meist führt sie zur Reduktion invasiver Maßnahmen, geringeren Beatmungsdrucken, niedrigeren inspiratorischen Sauerstoffkonzentrationen $\left(\mathrm{FiO}_{2}\right)$ und weniger Katecholamingaben. Natürlich wird man einen Patienten nicht ohne Not im hypoxämischen Bereich lassen; häufig ist aber eine Güterabwägung zwischen dem Risiko der invasiven Maßnahmen und dem Nutzen zu treffen.

In diesem Zusammenhang muss auch der heute sehr gebräuchliche Horowitz-Index oder auch Hypoxieindex gesehen werden, der in manchen Empfehlungen als Parameter für die Indikation zur Beatmung angesehen wird. Dieser errechnet sich aus der einfachen Beziehung des Sauerstoffpartialdruckes im arteriellen Blut zur inspiratorischen Sauerstofffraktion $\left(\mathrm{FiO}_{2}\right)$. Der Wert wird als Maß für die Schwere der Lungenschädigung bei ARDSPatienten herangezogen und dient häufig als klinischer Verlaufsparameter der ARDS-Therapie. Auch wenn in vielen Studien der Wert als ein Zielparameter eingesetzt wird, stellt er allenfalls eine grobe Abschätzung der Gasaustauschstörung dar. Der einfache Quotient aus Sauerstoffkonzentration in der Inspirationsluft und dem arteriellen Blut wird den komplexen pathophysiologischen Verhältnissen nicht gerecht, da er weder zwischen Diffusionsstörung und kritischer Shunterhöhung unterscheiden kann, noch als wesentliche Größe den applizierten Beatmungsdruck
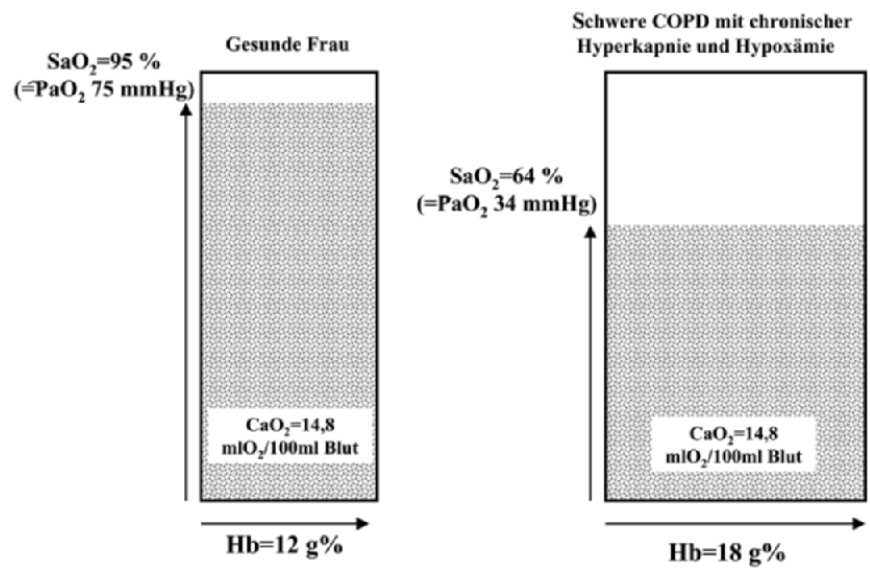

Abb. 4 Änderungen der Blutgase und der Sauerstoffsättigung, dargestellt an zwei Beispielen mit gleichem Sauerstoffgehalt. Links eine gesunde Frau mit Hämoglobin im unteren Normbereich und mit normaler Sauerstoffsättigung und -partialdruck. Rechts ein Patient mit schwerer COPD mit ventilatorischem Versagen (Hyperkapnie). Infolge der reaktiven Polyglobulie hat er den gleichen Sauerstoffgehalt wie ein Gesunder. Infolge der Hypoventilation ist jedoch die Sättigung und vor allen Dingen der Sauerstoffpartialdruck deutlich im pathologischen Bereich. Trotzdem haben beide Patienten das gleiche Sauerstoffangebot. Der Patient ist hypoxämisch, jedoch nicht hypoxisch.

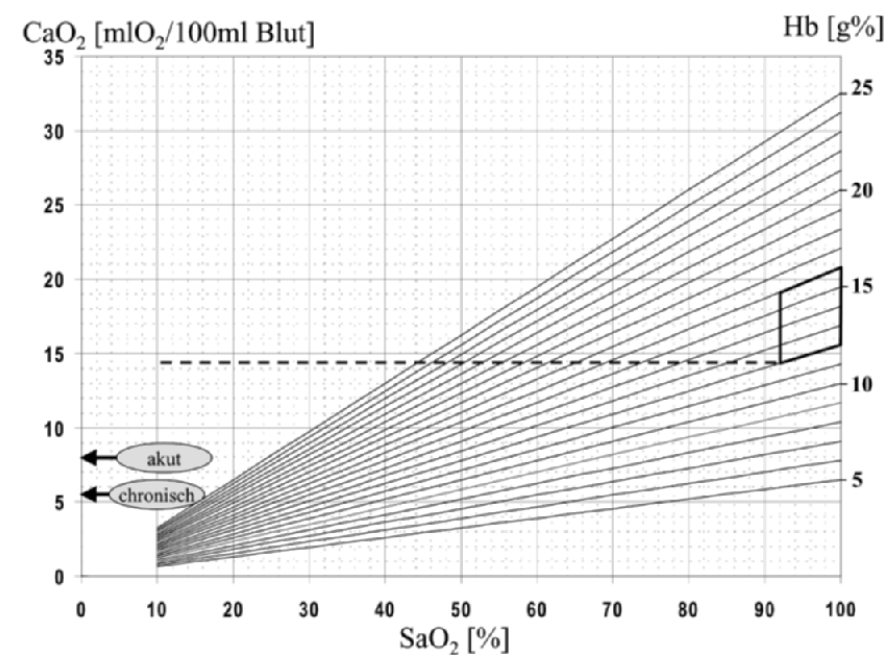

Abb. 5 Nomogramm zur Bestimmung des Sauerstoffgehalts aus Hämoglobin und Sauerstoffsättigung. Sollwerte nach anthropometrischen Daten für den Sauerstoffgehalt sind nicht verfügbar. Aus der Literatur ist zu entnehmen, dass der Grenzwert für einen akuten Abfall des Sauerstoffgehalts im Bereich von ca. $8 \mathrm{ml} \mathrm{O}_{2}$ pro $100 \mathrm{ml}$ Blut liegt. Bei chron. Hypoxämie liegt er durch Kompensationsvorgänge noch etwas tiefer. Die fett dargestellte Raute zeigt den Normwertbereich für Hämoglobin, Sauerstoffsättigung und Sauerstoffgehalt.

berücksichtigt. In der Praxis führt er daher eher zu Verwirrung, denn es ist ein großer Unterschied, ob z.B. ein Patient mit einer im Vordergrund stehenden Diffusionsstörung (der gut auf Sauerstoffgabe anspricht) mit einem Patienten mit deutlich erhöhtem Shuntvolumen (z.B. Pneumonie, der schlecht auf Sauerstoffgabe anspricht) verglichen wird. Ein einfaches Beispiel mag die Unterschiede aufzeigen. Wir haben bei Gesunden und stabilen Patienten mit COPD unterschiedliche $\mathrm{FiO}_{2}$ appliziert und den daraus errechneten Hypoxieindex dargestellt (Abb.6). Allein durch die Sauerstoffgabe erreichen viele Patienten einen deutlich patholo- 


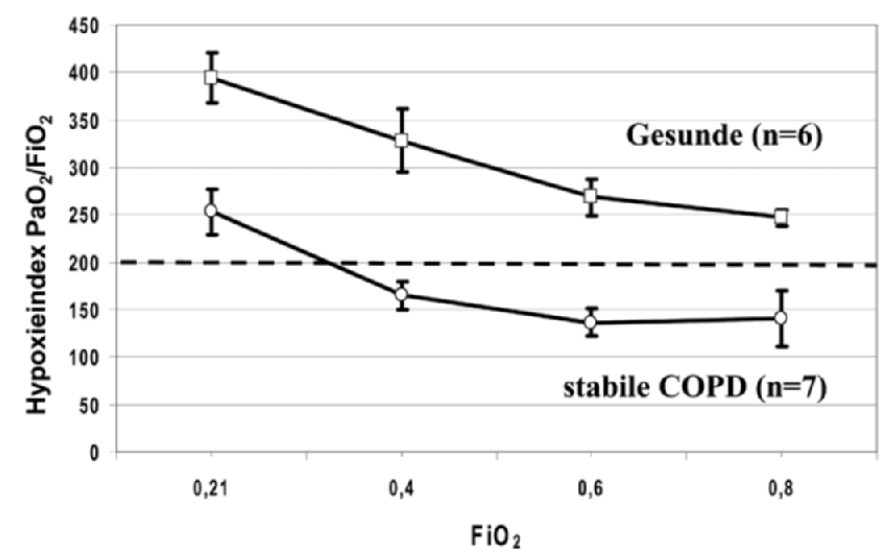

Abb. 6 Änderung des Hypoxieindex $\left(\mathrm{PaO}_{2} / \mathrm{FiO}_{2}\right)$ allein durch Sauerstoffgabe bei Patienten mit stabiler COPD und Gesunden. Man erkennt, dass infolge des Shuntvolumens allein durch Sauerstoffgabe bei stabilen COPD-Patienten der in manchen Leitlinien als Indikation zur Beatmung gesehene Grenzwert von 200 schnell unterschritten wird.

gischen Hypoxieindex von $\mathrm{PaO}_{2} / \mathrm{FiO}_{2}<200$, der in mehreren Leitlinien als Indikation für eine akute Beatmung angesehen wird. Hintergrund ist das erhöhte Shuntvolumen bei diesen Patienten. Trotz der Sauerstoffgabe wird der $\mathrm{PaO}_{2}$ im Shuntvolumen nicht beeinflusst, so dass der Gesamtsauerstoffpartialdruck über einen bestimmten Bereich nicht steigen kann.

\section{Atempumpeninsuffizienz}

Die Hauptindikation zur Beatmung ist das drohende oder bereits eingetretene Versagen der Atempumpe, sichtbar in einer Hypoventilation. In den Blutgasen drückt sie sich immer als Hyperkapnie aus. Konsekutiv entwickelt sich parallel immer eine Hypoxämie. Ziel der Beatmung sollte es sein, die Atempumpe nicht nur optimal zu entlasten, sondern auch einer Regeneration zuzuführen bzw. eine Hypotrophie der Muskulatur zu verhindern [51]. Naturgemäß darf dabei die zugrunde liegende Erkrankung, die für das Versagen der Atempumpe verantwortlich ist, nicht vergessen werden.

In Abb. 7 ist die grundsätzliche Problematik einer überlasteten quergestreiften Muskulatur dargestellt. Eine Hyperkapnie tritt immer dann auf, wenn die Atempumpe über den physiologischen Trainingsbereich hinaus belastet wird. Der Organismus kann der Grunderkrankung mit zunehmender Belastung der Atempumpe (z.B. fortschreitende COPD), nicht ausweichen und stellt auf eine kontrollierte Hypoventilation um, um ein sofortiges Versagen zu vermeiden. Der Trainingsgrad der Atemmuskulatur spielt bei kritischen Situationen auf der Intensivstation eine Rolle. Bei Patienten, die bereits eine trainierte Atemmuskulatur haben (z.B. chron. Obstruktion infolge COPD [52,53]), tritt ein Versagen später ein als bei Patienten, die eine untrainierte Muskulatur haben (z. B. akuter Asthmaanfall bei Patienten ohne vorausgegangene Obstruktion [54]). Dieses muss bei der Indikationsstellung zur Beatmung berücksichtigt werden. Wie in Abb. 7 dargestellt, ist die Therapie bei akut einsetzender oder sich akut verschlechternder Hyperkapnie immer die Entlastung. Biochemisch führt die Entlastung einer ermüdeten Muskulatur zu Repletion von Muskelglykogen, an dem der Muskel infolge Überbe-

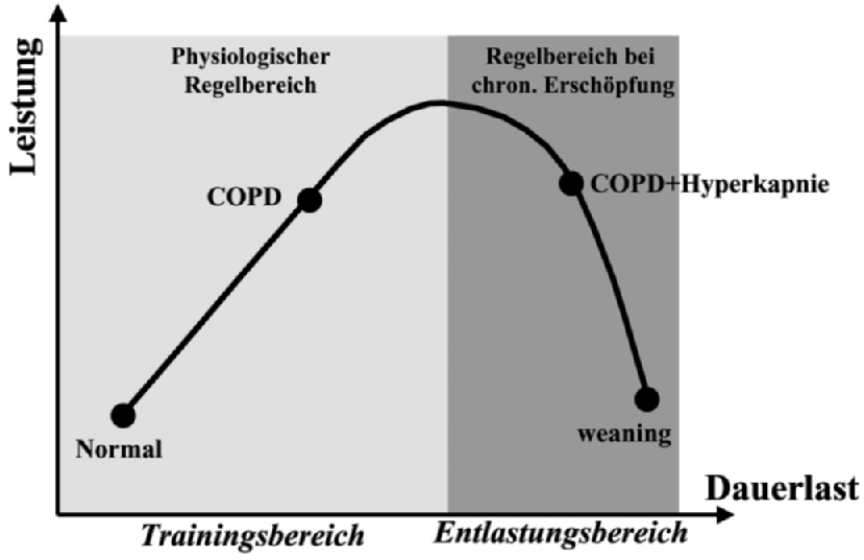

Abb. 7 Beziehung zwischen einer Dauerlast und der Leistungsentwicklung einer quergestreiften Muskulatur. Im Trainingsbereich kommt es durch Hypertrophie zu einer Zunahme der Leistung. Wird die Hypertrophiegrenze überschritten, kann bei unvermeidbarer Zunahme der Dauerlast (z.B. weitere Verschlechterung der COPD) ein Versagen der Muskulatur nur durch eine Leistungsabnahme kompensiert werden. Klinisch zeigt sich das als Hypoventilation, sichtbar in der Hyperkapnie. Aus der Abb. geht hervor, dass im Überlastbereich nur durch eine Entlastung der Muskulatur eine Leistungszunahme erreicht werden kann - im Gegensatz zum Trainingsbereich, wo es umgekehrt ist.

anspruchung verarmte [55 - 57]. Erste Maßnahme neben der Behandlung der Grunderkrankung und vor Beatmung ist die Gabe von Sauerstoff in niedrigeren Dosen, obwohl sie nur wenig bewirkt. Durch Verbesserung des Gasaustausches führt sie zu einer leichten Entlastung der Muskulatur, denn die Patienten regeln die Ventilation in dem Maße zurück, wie der $\mathrm{CaO}_{2}$ steigt (siehe Abb. 2). Allerdings liegt diese Entlastung nur im Bereich von ca. $10 \%$ (58). Bei intratrachealer Gabe ist die Entlastung der Atempumpe größer, da der Totraum mit ausgewaschen wird (ca. $20-30 \%)[59,60]$.

Ziel der Beatmung ist es also, nicht nur die akute Gasaustauschstörung infolge der Überlastung der Atempumpe zu korrigieren - was automatisch geschieht -, sondern auch die Atemmuskulatur direkt zu entlasten. Mit assistierter Ventilation ist das nur bedingt möglich (siehe unten). Natürlich ist die Situation anders, wenn Patienten über längere Zeiträume (beginnend vermutlich ab 2-3 Tage [61]) sediert und kontrolliert beatmet werden. Dann setzt rasch die Hypotrophie der Atmungsmuskulatur ein [51]. Um das zu verhindern, sollten Spontanatmungsphasen oder assistierte Beatmungsverfahren zwischengeschaltet werden.

In der Praxis gibt es nicht selten kombinierte Situationen mit gestörter Aufnahme des Sauerstoffs über das Lungenparenchym und gleichzeitiger Belastung der Atempumpe. Beispielsweise werden Patienten mit akuter Linksherzinsuffizienz kardial besser, wenn sie beatmet werden, obwohl sie zuvor keine Lungenerkrankung hatten. Eine akute Linksherzinsuffizienz (z.B. infolge Infarkt) führt durch den erhöhten pulmonalvenösen Druck zu einem Flüssigkeitstransport ins Interstitium des Lungenparenchyms. Dieses führt nicht nur zu einer verlängerten Diffusionsstrecke, sondern auch zu einer deutlichen Zunahme der Steifigkeit, was die Atemarbeit wiederum erhöht [62,63]. Deswegen 
muss ein relativ großer Teil der Perfusion - die infolge der akuten Herzinsuffizienz schon reduziert ist - in das belastete Zwerchfell fließen, um hier die Sauerstoffversorgung zu sichern. Wird der Patient sediert und kontrolliert beatmet, so geht die Sauerstoffaufnahme des Zwerchfells infolge fehlender Atemarbeit drastisch zurück, so dass intern Blut von dort auf das kranke Herz umverteilt werden kann [17]. Hinzu kommt, dass die Gesamtsauerstoffaufnahme $\left(\mathrm{VO}_{2}\right)$ etwas sinkt. Dieses führt weiterhin zur Entlastung des Herzens. Diese Veränderungen können aus der Gleichung in Abb. 2 abgeleitet werden.

Ein anderes Beispiel ist eine Pneumonie, die sich im Verlauf verschlechtert, dargestellt in Abb. 8. Hier führt am Anfang die Hypoxämie in den Blutgasen, ausgelöst durch Reduktion der Gasaustauschfläche infolge der Infiltrationen. Kompensatorisch kommt es zu einer Steigerung des Atemminutenvolumens, um den Sauerstofftransport zu sichern. Nimmt die Pneumonie weiter zu, so tritt im Verlauf eine Volumenbelastung der Atempumpe hinzu. Übersteigt diese die Belastungsreserve, so kommt es dann zur zusätzlichen ventilatorischen Insuffizienz. Der Verlauf entscheidet dann über den Beginn der Beatmung. Man sollte anfangen eine Pneumonie zu beatmen, wenn der Patient mit dem $\mathrm{PaCO}_{2}$ innerhalb kurzer Zeit ansteigt. Die Hypoxämie infolge der Pneumonie an sich ist noch keine Indikation zur Beatmung, da der Sauerstoffgehalt bei diesen Patienten üblicherweise noch nicht im kritischen Bereich liegt. Zumeist werden die Patienten jedoch bereits beatmet, so dass neben der Pneumonie ein zusätzlicher Dehnungsstress auf die Alveolen einwirkt. Da üblicherweise so weit beatmet wird, dass die Hypoxämie verschwindet, muss mit entsprechenden überproportionalen Volumina bzw. Drucken und hohen inspiratorischen Sauerstoffkonzentrationen gearbeitet werden, was wiederum die Entwicklung eines ARDS, bzw. VILI (Ventilator-Induced Lung Injury) begünstigt. Es darf auch nicht vergessen werden, dass die Ventilation über eine Maschine zu unphysiologischen Strömungsphänomenen bei der Inspiration führt. Insbesondere die basalen Anteile brauchen höhere Drucke, um sich zu entfalten, weil das Zwerchfell nicht aktiv heruntergezogen wird [64]. Versuche an Gesunden zeigen, dass unter Beatmung ca. ein Drittel mehr Atemminutenvolumen benötigt wird, um den gleichen Gasaustausch zu erzeugen wie bei Spontanatmung.

\section{Beatmung}

Eine Einarbeitung in die Beatmungsmedizin führt in der Regel zu einer gewissen Verwirrung infolge der zahlreichen angebotenen Beatmungsverfahren. Jedes Jahr kommen neue Begriffe hinzu. Eine zusammenfassende Übersicht mit kritischer Bewertung der einzelnen Verfahren wird man schwerlich finden, da insbesondere jede Disziplin und sogar manche Firmen ihre eigenen Begriffe bzw. Abkürzungen pflegen. Die Auswahl der Beatmungsverfahren wird auch durch die Krankheitsbilder bestimmt, die schwerpunktmäßig von den Fachgebieten bearbeitet werden. Beispielsweise wurde in den letzten Jahren ein großer Entwicklungsaufwand darauf verwandt, eine so genannte physiologisch-adaptierte Beatmung zu erreichen, bei der sich das angebotene Volumen dem Inspirationsdruck bzw. -fluss (Triggerreiz) des Patienten anpasst, die Proportional Assist Ventilation [65]. Ein weiterer Trend besteht darin, die Atemlage (FRC)

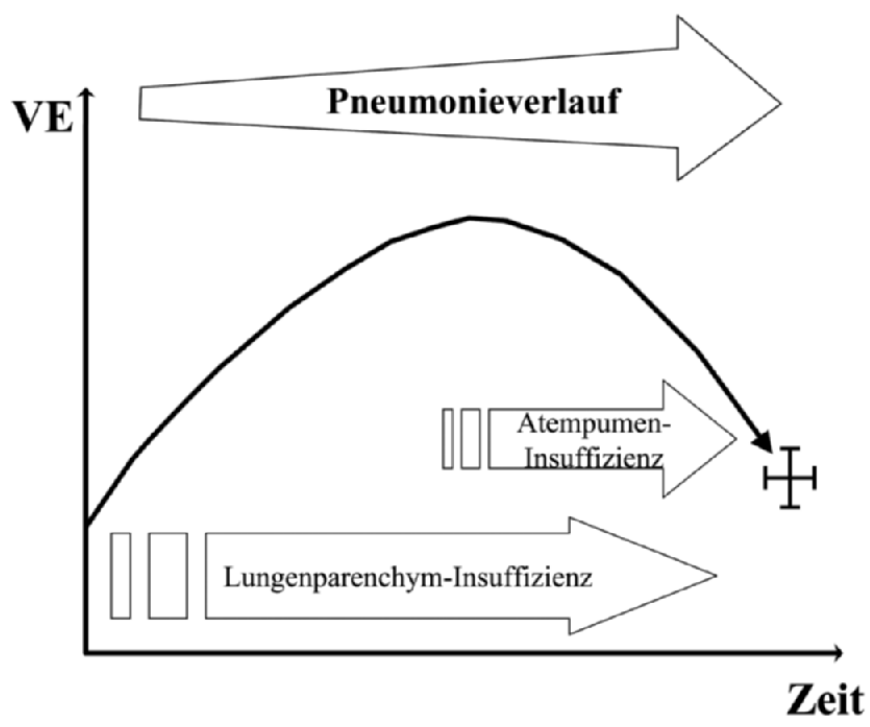

Abb. 8 Entwicklung einer ventilatorischen Insuffizienz aus einer hypoxischen am Beispiel einer Pneumonie bei Verschlechterung der Erkrankung. Anfangs kommt es durch die Hypoxämie zu einer Zunahme des Atemminutenvolumens, was die Blutgase stabilisiert. Erst wenn die Atemmuskulatur überlastet ist, kommt zu einer Hypoventilation und damit zur Entwicklung einer Hyperkapnie, um ein komplettes muskuläres Versagen zu vermeiden. Erst, wenn die ventilatorische Insuffizienz droht, ist die Indikation zur Beatmung gegeben.

des Patienten zu messen, um hier in einem möglichst günstigen Dehnungsbereich der Druck-Volumenkurve zu beatmen (lower/ upper inflation point) [66]. Es gibt auch aggressivere Verfahren, die den Gasaustausch dadurch zu verbessern versuchen, indem neue flüssige Sauerstoffträger wie Perfluorcarbon eingesetzt werden [67]. Berücksichtigt man die zuvor beschriebene Pathophysiologie des Sauerstofftransports, so stellt man fest, dass viele Probleme künstlich sind, weil als Zielgröße der Beatmung der $\mathrm{PaO}_{2}$ angesehen wird. Hierzu wird in manchen Lehrbüchern die Empfehlung ausgesprochen, die Beatmung so einzustellen, dass der $\mathrm{PaO}_{2}>80 \mathrm{~mm} \mathrm{Hg}$ und die $\mathrm{SaO}_{2}>95 \%$ liegen. Diese Empfehlung begünstigt nicht selten ein eigenes Krankheitsbild, die ventilatorinduzierte Lungenschädigung (Ventilator-Induced-LungInjury [VILI]). Die Lungenschädigung wird dabei durch hohe Beatmungsdrucke und hohe inspiratorische Sauerstoffkonzentrationen ausgelöst [4,61,68-71].

Nicht selten wird bei den Beatmungsgeräten zwischen solchen für Nicht-invasive-Beatmung (NIV) und Intubationsbeatmung unterschieden. Dies ist historisch bedingt, da anfangs Heimbeatmungsgeräte für die NIV auf der Intensivstation eingesetzt wurden. Diese verfügten (vernünftigerweise) nicht über die entsprechenden Alarmsysteme wie die Intensivbeatmungsgeräte. Heute sind die Übergänge zwischen den Beatmungsgeräten fließend, bzw. die Leistungsfähigkeit hat stark zugenommen. Eine NIV kann prinzipiell mit jedem Beatmungsgerät durchgeführt werden. Es handelt sich hierbei nur noch um einen anderen Beatmungszugang.

Die Berücksichtigung der $\mathrm{CaO}_{2}$ bei der Indikationsstellung zur Beatmung zeigt, dass sehr viel einfachere Beatmungsverfahren oft ausreichend sind. Hinzu kommt, dass bisher auf die Entlastung der Atemmuskulatur kaum Wert gelegt wurde. So ist z.B. 
das Prinzip der Proportional Assist Ventilation dem Entlastungsgedanken entgegengesetzt, denn der Patient bekommt dann viel Volumen, wenn er in der Lage ist, einen kräftigen Inspirationsfluss zu erzeugen. Bei einer erschöpften Atempumpe ist das aber genau umgekehrt, denn je erschöpfter die Atempumpe ist, desto geringer wird der Inspirationsfluss.

Die ideale Beatmungsform ist eine kontrollierte Beatmung ohne Spontanaktivität der Atempumpe mit zwischengeschalteten Spontanatmungsphasen, um einer Hypotrophie der Atmungsmuskulatur entgegenzuwirken. Hierbei sichert die Beatmungsmaschine zum einen die Ventilation und entlastet zum anderen die Atempumpe maximal, denn sie braucht nur die Sauerstoffaufnahme für den Ruhestoffwechsel [72-75] (Abb.9). Diese Form der Beatmung ist beim akuten Patienten praktisch nur durch entsprechende Sedation möglich. Eine zusätzliche Muskelrelaxation sollte wegen vieler anderer Probleme (insbesondere Neuropathie und Myopathie) vermieden werden [76], auch wenn sie den Gesamtsauerstoffverbrauch durch Rückgang des Muskelstoffwechsels $\left(\mathrm{VO}_{2}\right)$ nochmals senkt $[74,77]$. Allerdings scheint das nicht für die Sepsis zu gelten [78].

Eine kontrollierte Beatmung ist auch beim wachen Patienten möglich und wird meist bei elektiver häuslicher Beatmung in Deutschland durchgeführt. Hierzu ist aber eine entsprechende Trainingsphase von Stunden bis Tagen erforderlich. (Im Ausland steht dagegen für die häusliche Beatmung die assistierte Beatmungsform im Vordergrund.) Allerdings gilt das nicht für Patienten, die bereits an eine häusliche Beatmung (i. d. R. nicht-invasiv) adaptiert sind. Diese können bei Exazerbation der Grunderkrankung oft ohne Probleme auf der Intensivstation auch ohne (oder nur bei geringer) Sedation kontrolliert beatmet werden. Im Weaningprozess ist es zur weiteren Entlastung der Atmungsmuskulatur häufiger erforderlich, dass der Patient auf eine kontrollierte Beatmung bei wachem Bewusstsein mit zwischengeschalteten Spontanatmungsphasen eingestellt wird. Hierzu ist es notwendig, den Modus der Beatmungsmaschine etwa dem Patientenmuster anzupassen, wobei die Frequenz und das Atemzugvolumen gering höher liegen sollten [79].

In der Notfallsituation bei noch wachem Patienten ist die assistierte Beatmung der Standardfall (Abb. 10). Der Patient triggert die Maschine und bekommt dann ein entsprechendes Volumen bzw. eine Druckdifferenz angeboten, aus der das Volumen resultiert. Die meisten einfacheren Beatmungsgeräte arbeiten mit Volumen- oder mit Druckvorgaben, ohne wirkliche Endkontrolle des erreichten Beatmungsvolumens. Heute haben sich die Beatmungsgeräte mit Druckvorgabe durchgesetzt, da sie Druckspitzen vermeiden und schonender den Inspirationsfluss beginnen [80]. Größere Beatmungsgeräte haben heute intern die Möglichkeit, das Volumen (meist im Exspirationsschenkel) mitzumessen, um dann ggf. bei Nichterreichen eines Zielvolumens in einem gewissen vorgegebenen Bereich mit dem Druck nachzufahren.

Es darf aber nicht vergessen werden, dass bei der assistierten Beatmung der komplette Atemzyklus mitläuft, wenn auch nicht in vollem Umfange [73]. Messungen an Patienten mit chron. ventilatorischer Insuffizienz haben ergeben, dass der Sauerstoffverbrauch der Atempumpe bei assistierter Beatmung nur auf etwa $40 \%$ im Vergleich zur Spontanatmung reduziert ist $[72,81-83]$.

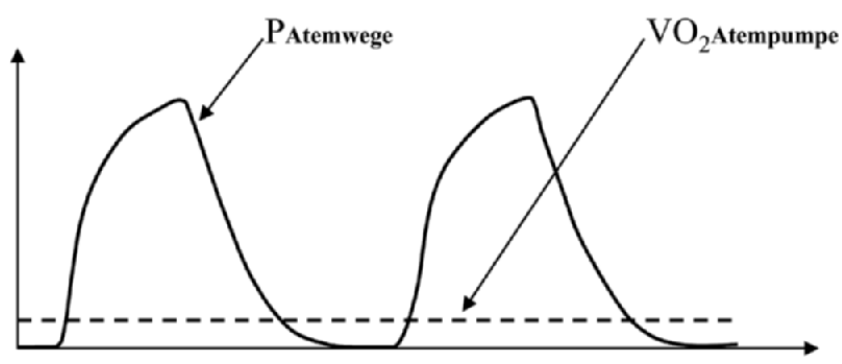

Zeit

Abb. 9 Kontrollierte Beatmung ohne Spontanaktivität (z. B. sedierter Patient oder Patient mit stabiler ventilatorischer Insuffizienz, der gelernt hat, seine Atemtätigkeit während der maschinellen Beatmung zu unterdrücken).

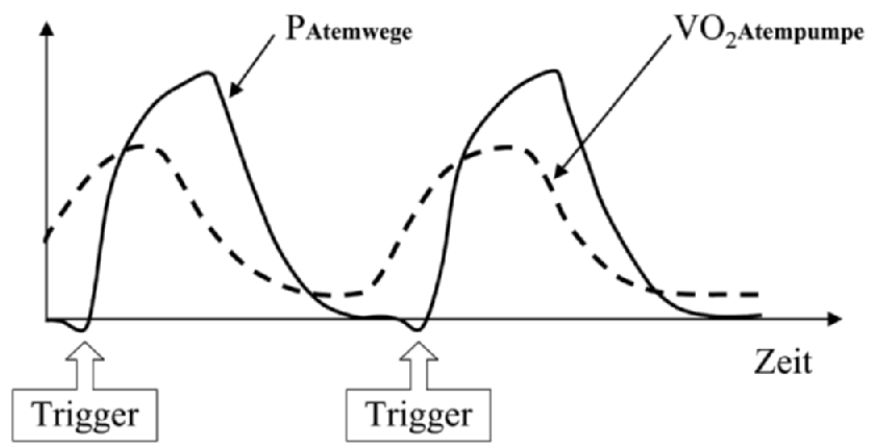

Abb. 10 Atemwegsdruck und Sauerstoffverbrauch der inspiratorischen Atemmuskulatur (Atempumpe) bei assistierter Beatmung. Hier triggert der Patient durch seinen eigenen Atemzyklus (mittels Unterdruck oder beginnendem Atemfluss) das Beatmungsgerät, was damit den Gasaustausch sichert. Der Atemzyklus läuft jedoch unterschwellig weiter, wenn auch mit geringerer Arbeit. Damit ist der Sauerstoffverbrauch der Atemmuskulatur und der Atempumpe deutlich höher als bei der kontrollierten Beatmung.

Macht man nach Besserung der Grunderkrankung bei assistierter Beatmung einen Spontanatmungsversuch, so hat die Atemmuskulatur naturgemäß nicht die Leistungsreserven wie nach kontrollierter Beatmung.

Ganz ungünstig ist der Versuch einer kontrollierten Beatmung bei Spontanatmung des Patienten, wenn unterschiedliche Atemfrequenzen bestehen bzw. der Atemzyklus der Maschine und der des Patienten nicht phasengleich verlaufen (Abb.11). In diesen Fällen atmet der Patient gegen die Maschine und muss zur Sicherung des Volumens zusätzliche Kraft aufbringen. Dadurch nimmt der Sauerstoffverbrauch der Atemmuskulatur noch zu. Diese Situation tritt nicht selten auf, wenn versucht wird, den Patient in der Aufwachphase in der kontrollierten Beatmung zu belassen. Eine permanente Kontrolle des Atemmusters mit Anpassung des Beatmungsgerätes ist dann erforderlich. Dazu benötigt das Personal entsprechende Schulung und Kenntnisse. Ansonsten wird - wie häufig - der Patient wieder sediert, was den Weaningprozess hinauszögert.

Wird ein Patient länger sediert und kontrolliert beatmet, so kommt es wie bei jeder quergestreiften Muskulatur relativ rasch zur Hypotrophie [51]. Diese beginnt bereits nach etwa 1-2 Ta- 


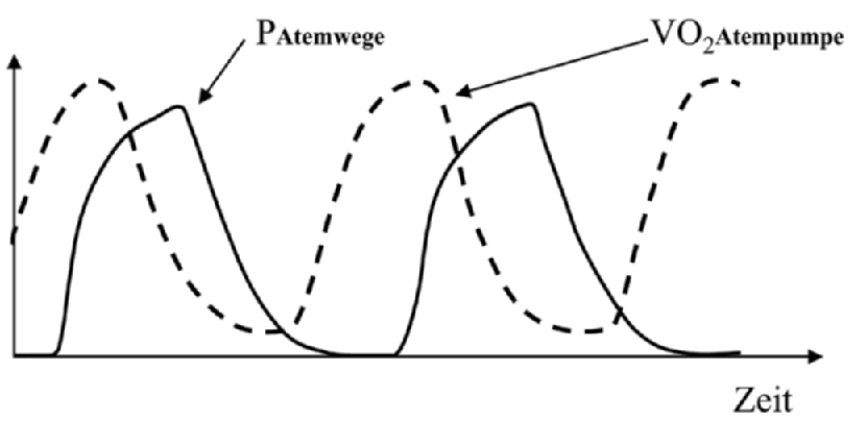

Abb. 11 Kontrollierte Beatmung mit Spontanaktivität. Ungünstigster Fall, da hier der Patient gegen die starre Atemfrequenz der Maschine atmen muss. Um das Atemminutenvolumen zu sichern, muss er z.T. aktiv gegenatmen, was den Sauerstoffverbrauch der Atempumpe im Vergleich zur Spontanatmung noch erhöht.

gen [61]. Es ist daher sinnvoll, immer wieder bei länger dauernder Beatmung die Sedierung zu unterbrechen und Spontanatmungsphasen bzw. assistierte Phasen zuzulassen, damit die Muskulatur nicht völlig atrophiert (VIDD = Ventilator-InducedDiaphragm-Dysfunction). Die tägliche Unterbrechung der Sedation hat darüber hinaus einen deutlich positiven Einfluss auf die Aufenthaltsdauer auf der Intensivstation [84]. Bei der NIV bleibt die ventilatorinduzierte Hypotrophie der Atemmuskulatur aus, da der Patient auch bei kontrollierter Beatmung immer wieder Spontanatmungsphasen zwischengeschaltet hat; z.B. um abzuhusten oder zu sprechen. Üblicherweise sollte die Unterbrechung der Sedation mit einem Entwöhnungsversuch verbunden werden. Ist die Muskulatur aber bereits atrophiert (was bei Weaningpatienten fast immer der Fall ist), so ist naturgemäß die Entlastungsstrategie noch wichtiger als bei nicht atrophierter Muskulatur, denn die Reserven sind entsprechend geringer. Deswegen muss nach ausreichender Entlastung schonend mit der Belastung begonnen werden. Legt man Daten aus der Sportmedizin zugrunde, so dauert es 6 - 8 Wochen, bis die durch die lange kontrollierte Beatmung entstandene Hypotrophie der Atemmuskulatur sich wieder zurückgebildet hat.

Abschließend soll ein typisches Beispiel die Unterschiede zwischen kontrollierter und assistierter Beatmung bei Patienten mit exazerbierter COPD nochmals verdeutlichen. In Abb. 12 ist ein Patient modellhaft dargestellt, dessen COPD exazerbiert. Infolge der Zunahme der Obstruktion kommt es zur erhöhten Atemarbeit mit entsprechend vermehrter Sauerstoffaufnahme. Die Atempumpe wird überlastet, und es entwickelt sich konsekutiv eine Hypoventilation mit Hyperkapnie. Der Patient muss einige Tage beatmet werden, um die kritische Phase zu überstehen. Bei assistierter Beatmung fällt der $\mathrm{PaCO}_{2}$ im gleichen $\mathrm{Maße}$ wie bei kontrollierter Beatmung; die Sauerstoffaufnahme der Atempumpe $\left(\mathrm{VO}_{2}\right)$ ist jedoch deutlich höher.

Wird der Patient in dieser Zeit sediert und kontrolliert beatmet (Abb. 13) oder er lernt ohne Sedation bei kontrolliertem Modus die Spontanatmung zu unterdrücken, so rekompensiert die Atempumpe rascher, und der Patient kann sich am Ende der Beatmung infolge der stärkeren Rekompensation ein höheres Atemminutenvolumen leisten. Dies ist dann sichtbar an einem niedrigeren $\mathrm{pCO}_{2}$ am Ende der anschließenden Spontanatmungsphase.

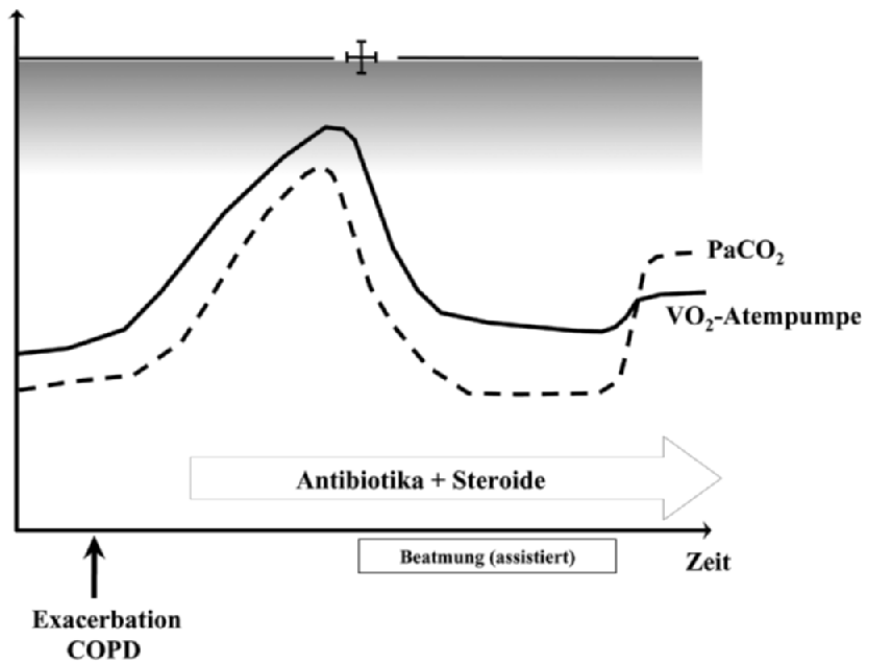

Abb. $12 \mathrm{PaCO}_{2}$-Verlauf und Sauerstoffverbrauch der Atempumpe, dargestellt an einer Exazerbation einer COPD mit assistierter und kontrollierter (Abb. 13) Beatmung. Infolge der Zunahme der Obstruktion erhöht sich der Sauerstoffverbrauch der Atempumpe. Um ein lebensbedrohliches Versagen zu vermeiden, kommt es zur Gegenregulation mittels Hypoventilation, sichtbar an einer Hyperkapnie. Wenn die Therapie der Exazerbation mit Antibiotika und Steroiden nicht ausreicht und die Hyperkapnie weiter zunimmt, muss der Patient beatmet werden. Beim assistierten Modus kommt es zwar zu einer deutlichen Besserung des Gasaustausches durch die normalisierte Ventilation; infolge der unterschwellig weiterlaufenden Eigenatmung kommt es nur zu einer mäßigen Entlastung des Sauerstoffverbrauchs der Atempumpe, die das limitierende Organ darstellt.

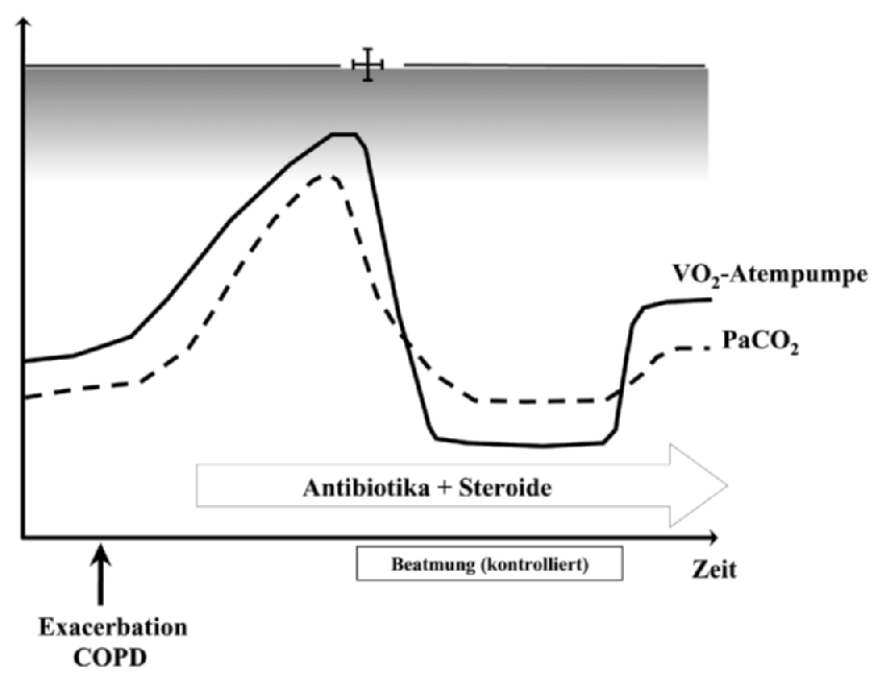

Abb. 13 Gleiche Situation wie Abb. 12 mit dem Unterschied der kontrollierten Beatmung mit der Folge einer deutlichen Reduktion des Sauerstoffverbrauchs der Atempumpe. Diese hat dadurch eine deutlich bessere Erholungsmöglichkeit, was sich in einem niedrigeren $\mathrm{PaCO}_{2}$-Wert nach Abschluss der Beatmung widerspiegelt. Die Rekompensation erfolgt damit deutlich schneller.

Zusammenfassend hat sich in der Beatmung in den letzten Jahren durch Berücksichtung der Pathophysiologie der belasteten Atempumpe ein entscheidender Wandel vollzogen. Dies wurde ausgelöst durch die Etablierung und Verbreitung der NIV. Die weitere Berücksichtigung des damit zusammenhängenden Gastransportes in der Gesamtschau steht erst am Anfang. 
${ }^{26}$ Gattinoni L, Tognoni G, Pesenti A et al. Effect of prone positioning on the survival of patients with acute respiratory failure. N Engl J Med 2001; 345: $568-573$

${ }^{1}$ Esteban A, Anzueto A, Frutos F et al. Mechanical Ventilation International Study Group. Characteristics and outcomes in adult patients receiving mechanical ventilation: a 28-day international study. JAMA 2002; 16 (287): $345-355$

2 Tobin MJ. Advances in mechanical ventilation. N Engl J Med 2001; 344: 1986 - 1996

${ }^{3}$ Tobin MJ. Mechanical ventilation. N Engl J Med 1994; 330: 1056 - 1061

${ }^{4}$ No authors listed. Ventilation with lower tidal volumes as compared with traditional tidal volumes for acute lung injury and the acute respiratory distress syndrome. The Acute Respiratory Distress Syndrome Network. N Engl J Med 2000; 342: 1301 - 1308

${ }^{5}$ Denis D, Fayon MJ, Berger $\mathrm{P}$ et al. Prolonged moderate hyperoxia induces hyperresponsiveness and airway inflammation in newborn rats. Pediatr Res 2001; 50: 515 - 519

${ }^{6}$ Ferrara TB, Fox RB. Effects of oxygen toxicity on cuprolinic blue-stained proteoglycans in alveolar basement membranes. Am J Respir Cell Mol Biol 1992; 6: 219-224

${ }^{7}$ Pagano A, Barazzone-Argiroffo C. Alveolar cell death in hyperoxia-induced lung injury. Ann N Y Acad Sci 2003; 1010: 405 - 416

${ }^{8}$ Mencke T, Echternach M, Kleinschmidt S et al. Laryngeal morbidity and quality of tracheal intubation: a randomized controlled trial. Anesthesiology 2003; 98: 1049-1056

${ }^{9}$ Sue RD, Susanto I. Long-term complications of artificial airways. Clin Chest Med 2003; 24: 457-471

${ }^{10}$ Keller C, Brimacombe J. Bronchial mucus transport velocity in paralyzed anesthetized patients: a comparison of the laryngeal mask airway and cuffed tracheal tube. Anesth Analg 1998; 86: 1280-1282

${ }^{11}$ Konrad F, Schreiber T, Brecht-Kraus D et al. Mucociliary transport in ICU patients. Chest 1994; 105: 237-241

12 Sanada Y, Kojima Y, Fonkalsrud EW. Injury of cilia induced by tracheal tube cuffs. Surg Gynecol Obstet 1982; 154: 648-652

${ }^{13}$ Fellahi JL, Valtier B, Beauchet A et al. Does positive end-expiratory pressure ventilation improve left ventricular function? A comparative study by transesophageal echocardiography in cardiac and noncardiac patients. Chest 1998; 114: 556-562

${ }^{14}$ Meurice JC, Mergy J, Rostykus C et al. A trial arrhythmia as a complication of nasal CPAP. Chest 1992; 102: 640-642

15 Tuxen DV. Detrimental effects of positive end-expiratory pressure during controlled mechanical ventilation of patients with severe airflow obstruction. Am Rev Respir Dis 1989; 140: 5 -9

${ }^{16}$ Hussain SN, Roussos C. Distribution of respiratory muscle and organ blood flow during endotoxic shock in dogs. J Appl Physiol 1985; 59: $1802-1808$

17 Viires N, Sillye G, Aubier M et al. Regional blood flow distribution in dog during induced hypotension and low cardiac output. Spontaneous breathing versus artificial ventilation. J Clin Invest 1983; 72: 935-947

${ }^{18}$ Agusti AG, Rodriguez-Roisin R, Roca J et al. Oxyhemoglobin affinity in patients with chronic obstructive pulmonary disease and acute respiratory failure: role of mechanical ventilation. Crit Care Med 1986; 14: $610-613$

${ }^{19}$ Thorens JB, Jolliet P, Ritz M et al. Effects of rapid permissive hypercapnia on hemodynamics, gas exchange, and oxygen transport and consumption during mechanical ventilation for the acute respiratory distress syndrome. Intensive Care Med 1996; 22: 182-191

${ }^{20}$ Butterick CJ, Williams DA, Boxer LA et al. Changes in energy metabolism, structure and function in alveolar macrophages under anaerobic conditions. Br J Haematol 1981; 48: 523-532

${ }^{21}$ Simon LM, Robin ED, Phillips JR et al. Enzymatic basis for bioenergetic differences of alveolar versus peritoneal macrophages and enzyme regulation by molecular $\mathrm{O}_{2}$. J Clin Invest 1977; 59: $443-448$

22 Terrados N, Jansson E, Sylven C et al. Is hypoxia a stimulus for synthesis of oxidative enzymes and myoglobin? J Appl Physiol 1990; 68: $2369-2372$

${ }^{23}$ Cartee GD, Douen AG, Ramlal T et al. Stimulation of glucose transport in skeletal muscle by hypoxia. J Appl Physiol 1991; 70: 1593-1600

${ }^{24}$ Dery MA, Michaud MD, Richard DE. Hypoxia-inducible factor 1 : regulation by hypoxic and non-hypoxic activators. Int J Biochem Cell Biol 2005; 37: 535-540

${ }^{25}$ Xia Y, Warshaw JB, Haddad GG. Effect of chronic hypoxia on glucose transporters in heart and skeletal muscle of immature and adult rats. Am J Physiol 1997; 273: R1734-1741

${ }^{27}$ British Thoracic Society Standards of Care Committee. Non-invasive ventilation in acute respiratory failure. Thorax 2002; 57: 192-211

${ }^{28}$ Begin P, Grassino A. Inspiratory muscle dysfunction and chronic hypercapnia in chronic obstructive pulmonary disease. Am Rev Respir Dis 1991; 143: $905-912$

${ }^{29}$ Begin P, Mathieu J, Almirall J et al. Relationship between chronic hypercapnia and inspiratory-muscle weakness in myotonic dystrophy. Am J Respir Crit Care Med 1997; 156: 133-139

${ }^{30}$ Gorini M, Misuri G, Corrado A et al. Breathing pattern and carbon dioxide retention in severe chronic obstructive pulmonary disease. Thorax 1996; 51: 677-683

${ }^{31}$ Köhler D, Schönhofer B. Apnoe-hypopnoe. Eine oder zwei Entitäten? Pneumologie 1998; 52: $311-318$

${ }^{32}$ Leach RM, Treacher DF. The pulmonary physician in critical care * 2 : oxygen delivery and consumption in the critically ill. Thorax 2002; 57: $170-177$

${ }^{33}$ Hayes MA, Timmins AC, Yau EH et al. Elevation of systemic oxygen delivery in the treatment of critically ill patients. N Engl J Med 1994; 330: $1717-1722$

${ }^{34}$ Weg JG. Oxygen transport in adult respiratory distress syndrome and other acute circulatory problems: relationship of oxygen delivery and oxygen consumption. Crit Care Med 1991; 19: 650-657

${ }^{35}$ Hayes MA, Yau EH, Timmins AC et al. Response of critically ill patients to treatment aimed at achieving supranormal oxygen delivery and consumption. Relationship to outcome. Chest 1993; 103: 886-895

${ }^{36}$ Hebert PC, Linden P van der, Biro G et al. Physiologic aspects of anemia. Crit Care Clin 2004; 20: 187-212

37 Schonhofer B, Wenzel M, Geibel M et al. Blood transfusion and lung function in chronically anemic patients with severe chronic obstructive pulmonary disease. Crit Care Med 1998; 26: 1824-1828

${ }^{38}$ Schonhofer B, Bohrer H, Kohler D. Blood transfusion facilitating difficult weaning from the ventilator. Anaesthesia 1998; 53: 181 - 184

${ }^{39}$ Greif R, Akca O, Horn EP et al. Supplemental perioperative oxygen to reduce the incidence of surgical-wound infection. Outcomes Research Group. N Engl J Med 2000; 342: 161 - 167

40 Bernstein D, Teitel DF. Myocardial and systemic oxygenation during severe hypoxemia in ventilated lambs. Am J Physiol 1990; 258: H1856- 1864

${ }^{41}$ Borgia JF, Horvath SM. Effects of acute prolonged hypoxia on cardiovascular dynamics in dogs. J Appl Physiol 1977; 43: 784-789

42 Mazer CD, Stanley WC, Hickey RF et al. Myocardial metabolism during hypoxia: maintained lactate oxidation during increased glycolysis. Metabolism 1990; 39: 913 -918

43 Taylor PM. Effects of hypoxia on endocrine and metabolic responses to anaesthesia in ponies. Res Vet Sci 1999; 66: 39-44

${ }^{44}$ Todd MM, Wu B, Maktabi M et al. Cerebral blood flow and oxygen delivery during hypoxemia and hemodilution: role of arterial oxygen content. Am J Physiol 1994; 267: H2025-2031

${ }^{45}$ Grubbstrom J, Berglund B, Kaijser L. Myocardial oxygen supply and lactate metabolism during marked arterial hypoxaemia. Acta Physiol Scand 1993; 149: $303-310$

${ }^{46}$ Kaijser L, Grubbstrom J, Berglund B. Myocardial lactate release during prolonged exercise under hypoxaemia. Acta Physiol Scand 1993; 149: $427-433$

${ }^{47}$ Gupta GD, Jain SK, Dasgupta SR. Haemodynamic changes with blood transfusion in chronic severe anaemia. Acta Cardiol 1977; 32: 25 - 39

${ }^{48}$ Roach RC, Koskolou MD, Calbet JA et al. Arterial $\mathrm{O}_{2}$ content and tension in regulation of cardiac output and leg blood flow during exercise in humans. Am J Physiol 1999; 276: H438 - 445

${ }^{49}$ Sutton JR, Reeves JT, Wagner PD et al. Operation Everest II: oxygen transport during exercise at extreme simulated altitude. J Appl Physiol 1988 Apr; 64 (4): 1309-1321

${ }^{50}$ West JB, Boyer SJ, Graber DJ et al. Maximal exercise at extreme altitudes on Mount Everest. J Appl Physiol 1983; 55: 688 - 698

51 Gayan-Ramirez G, Decramer M. Effects of mechanical ventilation on diaphragm function and biology. Eur Respir J 2002; 20: 1579-1586

52 Enright S, Chatham K, Ionescu AA et al. Inspiratory muscle training improves lung function and exercise capacity in adults with cystic fibrosis. Chest 2004; 126: $405-411$

${ }^{53}$ Newell SZ, McKenzie DK, Gandevia SC. Inspiratory and skeletal muscle strength and endurance and diaphragmatic activation in patients with chronic airflow limitation. Thorax 1989; 44: $903-912$ 
${ }^{54}$ de Bruin PF, Ueki J, Watson A et al. Size and strength of the respiratory and quadriceps muscles in patients with chronic asthma. Eur Respir J 1997; 10: $59-64$

${ }^{55}$ Connett RJ, Honig CR, Gayeski TE et al. Defining hypoxia: a systems view of $\mathrm{VO}_{2}$, glycolysis, energetics, and intracellular $\mathrm{PO}_{2}$.J Appl Physiol 1990; 68: $833-842$

${ }^{56}$ Ferguson GT, Irvin CG, Cherniack RM. Relationship of diaphragm glycogen, lactate, and function to respiratory failure. Am Rev Respir Dis 1990; 141: $926-932$

${ }^{57}$ Lockhat D, Roussos C, Ianuzzo CD. Metabolite changes in the loaded hypoperfused and failing diaphragm. J Appl Physiol 1988; 65: $1563-1571$

${ }^{58}$ Marczak M, Pokorski M. Oxygen breathing and ventilation. J Physiol Pharmacol 2004; 55: 127-134

${ }^{59}$ Benditt Jr J, Make BJ. Transtracheal oxygen decreases inspired minute ventilation. Am Rev Respir Dis 1989; 139: 627-631

${ }^{60}$ Benditt J, Pollock M, Roa J et al. Transtracheal delivery of gas decreases the oxygen cost of breathing. Am Rev Respir Dis 1993; 147: $1207-1210$

${ }^{61}$ Shanely RA, Gammeren D van, Deruisseau KC et al. Mechanical ventilation depresses protein synthesis in the rat diaphragm. Am J Respir Crit Care Med 2004; 170: $994-999$

${ }^{62}$ Agostoni P, Cattadori G, Bianchi M et al. Exercise-induced pulmonary edema in heart failure. Circulation 2003; 108: 2666-2671

${ }^{63}$ Nava S, Larovere MT, Fanfulla F et al. Orthopnea and inspiratory effort in chronic heart failure patients. Respir Med 2003; 97: 647-653

${ }^{64}$ Beydon L, Cinotti L, Rekik N et al. Changes in the distribution of ventilation and perfusion associated with separation from mechanical ventilation in patients with obstructive pulmonary disease. Anesthesiology $1991 ; 75: 730-738$

65 Ambrosino N, Rossi A. Proportional assist ventilation (PAV): a significant advance or a futile struggle between logic and practice? Thorax 2002; 57: $272-276$

${ }^{66}$ Maggiore SM, Richard JC, Brochard L. What has been learnt from P/V curves in patients with acute lung injury/acute respiratory distress syndrome. Eur Respir J Suppl 2003; 42: 22s-26s

67 Papo MC, Paczan PR, Fuhrman BP et al. Perfluorocarbon-associated gas exchange improves oxygenation, lung mechanics, and survival in a model of adult respiratory distress syndrome. Crit Care Med 1996; 24: $466-474$

${ }^{68}$ Frank JA, Matthay MA. Science review: mechanisms of ventilator-induced injury. Crit Care 2003Jun; 7: 233-241

${ }^{69}$ Gattinoni L, Caironi P, Carlesso E. How to ventilate patients with acute lung injury and acute respiratory distress syndrome. Curr Opin Crit Care 2005; 11: $69-76$
${ }^{70}$ Lionetti V, Recchia FA, Ranieri VM. Overview of ventilator-induced lung injury mechanisms. Curr Opin Crit Care 2005; 11: 82 - 86

${ }^{71}$ Ricard JD, Dreyfuss D, Saumon G. Ventilator-induced lung injury. Curr Opin Crit Care 2002; 8: $12-20$

72 Cinnella G, Conti G, Lofaso F et al. Effects of assisted ventilation on the work of breathing: volume-controlled versus pressure-controlled ventilation. Am J Respir Crit Care Med 1996; 153: 1025-1033

${ }^{73}$ Hubmayr RD, Loosbrock LM, Gillespie DJ et al. Oxygen uptake during weaning from mechanical ventilation. Chest 1988; 94: 1148-1155

${ }^{74}$ Manthous CA, Hall JB, Kushner R et al. The effect of mechanical ventilation on oxygen consumption in critically ill patients. Am J Respir Crit Care Med 1995; 151: 210-214

75 Rice AJ, Nakayama HC, Haverkamp HC et al. Controlled versus assisted mechanical ventilation effects on respiratory motor output in sleeping humans. Am J Respir Crit Care Med 2003; 168: 92 - 101

${ }^{76}$ Murphy GS, Vender JS. Neuromuscular-blocking drugs. Use and misuse in the intensive care unit. Crit Care Clin 2001; 17: 925-942

${ }^{77}$ Vernon DD, Witte MK. Effect of neuromuscular blockade on oxygen consumption and energy expenditure in sedated, mechanically ventilated children. Crit Care Med 2000; 28: 1569-1571

${ }^{78}$ Freebairn RC, Derrick J, Gomersall CD et al. Oxygen delivery, oxygen consumption, and gastric intramucosal $\mathrm{pH}$ are not improved by a computer-controlled, closed-loop, vecuronium infusion in severe sepsis and septic shock. Crit Care Med 1997; 25: $72-77$

${ }^{79}$ Schönhofer B, Köhler D. Ventilatorische Insuffizienz und hyperkapnische Kompensation infolge chronisch belasteter Atempumpe. Dtsch Med Wochenschr 1994 Sep 9; 119 (36): 1209-1214

${ }^{80}$ Kallet RH, Campbell AR, Alonso JA et al. The effects of pressure control versus volume control assisted ventilation on patient work of breathing in acute lung injury and acute respiratory distress syndrome. Respir Care 2000; 45: 1085-1096

${ }^{81}$ Leung P, Jubran A, Tobin MJ. Comparison of assisted ventilator modes on triggering, patient effort, and dyspnea. Am J Respir Crit Care Med 1997; 155: 1940 - 1948

82 Swinamer DL, Fedoruk LM, Jones RL et al. Energy expenditure associated with CPAP and T-piece spontaneous ventilatory trials. Changes following prolonged mechanical ventilation. Chest 1989; 96: $867-872$

${ }^{83}$ Rasche K, Laier-Groeneveld G, Weyland W et al. Sauerstoffverbrauch der Atemmuskulatur unter kontrollierter bzw. assistierter Beatmung bei Patienten mit chronischer Ateminsuffizienz. Med Klin 1994; 89: $43-46$

${ }^{84}$ Kress JP, Pohlman AS, O'Connor MF et al. Daily interruption of sedative infusions in critically ill patients undergoing mechanical ventilation. N Engl J Med 2000; 342: 1471 - 1477 